\title{
ELECTRICAL PHOTO-ENGRAVING
}

$\mathrm{M}^{\mathrm{s}}$ R. T. THORNE BAKER describes in the Electrical Review of August 23 a new phase of facsimile reproduction for newspaper printing. A study of the literature of early telegraphic transmission of pictures shows that inventors were directed long ago towards the possibilities of receiving a telegraphed image directly upon a soft metal sheet, a cutting tool (operated electro-magnetically by the receiving currents) making an intaglio image which could be printed forthwith. Recent literature on the subject shows that the general opinion nowadays is that it is more profitable to transmit a continuoustone image from which the screen picture for reproduction can be made by the ordinary methods.

It has been proved that by transmitting a picture by photo-electric scanning to another cylinder rigidly attached to the scanning cylinder, a new means has been established of reproducing photographs which eliminates the camera. This method has opened up a most important field which is likely to have far-reaching effects upon the photo-engraving industry. It applies equally to black-and-white work and colour, and offers, for the first time in photographic reproduction history, a means of reproducing almost perfect copies of any original with little of the 'retouching' and 'fine etching' which to-day makes photo-engraving unnecessarily costly.

In the usual method, the original photograph is attached to the drum of the sending instrument and the surface is scanned by a beam of light which is made to trace a spiral path round the drum, the light after refraction by the picture tones being reflected back on the photo-cell. The amplified output currents from the cell are transmitted to the distant receiving instrument, where they operate a light-valve which exposes a sensitive film, or makes an electromagnetically actuated stencil mark the paper through a sheet of ordinary carbon paper. An alternative method is to use the received currents to operate a high potential glow lamp, the rays of which are concentrated through a tiny aperture upon the surface of the sensitive paper.

Recent sensitometric investigations have indicated the exact losses in tone rendering when making half- tone reproductions by ordinary methods of photoengraving, in both the high-lights and shadows of the image. In the ordinary way these are overcome by retouching the original, skilled manipulation of the negative, and fine etching of the metal block. It is possible to bias the currents from the scanning unit so as to put back, as it were, what is lost in photo-mechanical reproduction processes. Selecting suitable portions of the characteristic curves of the valves used in amplification, and using the photocells themselves under selected conditions, makes it possible to add to or take away from the tones of the facsimile image at any desired densities.

One method of obtaining this result is embodied in a newspaper engraving unit and made by the author for a provincial American newspaper. A transparent film is attached to a drum, actually a glass cylinder, and light from a lamp is focused by a lens, so that the rays come to the periphery of the cylinder. The light beam, after passing through the photographic original, is spread by a negative lens so as to fall on three photo-cells $P_{1}, P_{2}$ and $P_{3}$. Cell $P_{2}$ gives a straight line reproduction of the image over most of the inner tones. Cell $P_{1}$ is so arranged electrically that it records the high-light tones only, and adds density to the upper part of the reproduction curve in the manner indicated by Tritton and Wilson, in the Photographic Journal of June 1939. Cell $P_{3}$ similarly adds densities in the shadows. The currents from the three cells after amplification are mixed and made to operate a glow lamp, to the rays which the receiving film is exposed. In this way a biased reproduction is obtained, which when received by the half-tone screen in the process camera, yields a half-tone negative from which a block can be made that will give 'perfect' tone rendering without hard work. The Trenton Times (New Jersey, U.S.A.), which has adopted the system, prints high-speed offset lithographic four-colour pictures with 120 -line screen at 45,000 copies per hour, which is something unique at present in newspaper printing. The primary purpose of the system is to speed up the making of half-tones for newspaper printing.

\section{THE THEORY OF ADSORPTION ON SQUARE LATTICES}

\author{
BY DR. J. K. ROBERTS, \\ Department of Colloid Science, Cambridge
}

$\mathrm{I}^{\mathrm{N}}$ a recent paper L. Tonks ${ }^{1}$ has criticized the application of the Bethe method to the solution of problems in adsorption. I. Langmuir ${ }^{2}$ had earlier mentioned the criticism and supported it. I have already given an answer ${ }^{3}$ to Langmuir's remarks so far as was possible before the appearance of the paper by Tonks.

We are concerned with the adsorption on a square lattice of particles so large that, if one site is occupied, adsorption cannot take place on the four surrounding sites. Thus, if a particle is on site $o$ in Fig. 1, adsorption on the four sites marked $a$ is excluded.

Bethe's method depends on the selection of a small group of sites on which all possible configurations of adsorbed particles are considered, and suitable parameters are introduced to take account of the effect of the particles outside the group on those within it, and vice versa. Thus in Fig. 1, if we call site $o$ the central site, and the sites marked $a, b, c$ first shell, second shell and third shell sites respectively, 